\title{
Estimation of Propagation Characteristics along Random Rough Surface for Sensor Networks
}

\author{
Kazunori Uchida and Junichi Honda \\ Fukuoka Institute of Technology \\ Japan
}

\section{Introduction}

The main focus in the development of wireless communications engineering is providing higher data rates, using lower transmission power, and maintaining quality of services in complicated physical environments, such as an urban area with high-rise buildings, a randomly profiled terrestrial ground and so on. In order to achieve these goals, there has been substantial progress in the development of low-power circuits, digital algorithms for modulation and coding, networking controls, and circuit simulators in recent years [Aryanfar,2007]. However, insufficient improvement has been made in wireless channel modeling which is one of the most basic and significant engineering problems corresponding to the physical layer of the OSI model.

Recently, the sensor network technologies have attracted many researchers' interest especially in the fields of wireless communications engineering as well as in the fields of sensor engineering. The sensor devices are usually located on the terrestrial surfaces such as dessert, hilly terrain, forest, sea surface and so on, of which profiles are considered to be statistically random. In this context, it is very important to investigate the propagation characteristics of electromagnetic waves traveling along random rough surfaces (RRSs) and construct an efficient as well as reliable sensor network over terrestrial grounds with RRS-like profiles [Uchida,2007], [Uchida,2008], [Uchida,2009], [Honda,2010].

In the early years of our investigations, we applied the finite volume time domain (FVTD) method to estimate the electromagnetic propagation characteristics along one-dimensional (1D) RRSs [Honda,2006], [Uchida,2007]. The FVTD method, however, requires too much computer memory and computation time to deal with relatively long RRSs necessary for a sensor network in the realistic situation. To overcome this difficulty, we have introduced the discrete ray tracing method (DRTM) based on the theory of geometrical optics, and we can now deal with considerably long RRSs in comparison with the operating wavelength. The merit of using DRTM is that we can treat very long RRSs compared with the wavelength without much computer memory nor computation time. Thus, the DRTM has become one of the most powerful tools in order to numerically analyze the long-distance propagation characteristics of electromagnetic waves traveling along RRSs [Uchida,2008], [Uchida,2009], [Honda,2010]. In this chapter, we discuss the distance characteristics of electromagnetic waves propagating along homogeneous RRSs which are described statistically in terms of the two parameters, that is, height deviation $h$ and correlation length $c \ell$. The distance characteristics of propagation are estimated by introducing an amplitude weighting factor $\alpha$ for field amplitude, an 
order $\beta$ for an equivalent propagation distance, and a distance correction factor $\gamma$. The order yields an equivalent distance indicating the distance to the $\beta$-th power. The order was introduced by Hata successfully as an empirical formula for the propagation characteristics in the urban and suburban areas [Hata,1980]. In the present formulations, we determine these parameters numerically by using the least square method. Once these parameters are determined for one type of RRSs, we can easily estimate the radio communication distance between two sensors distributed on RRSs, provided the input power of a source antenna and the minimum detectable electric field intensity of a receiver are specified.

The contents of the present chapter are described as follows. Section 1 is the introduction of this chapter, and the background of this research is denoted. Section 2 discusses the statistical properties of 1D RRSs and the convolution method is introduced for RRS generation. Section 3 discusses DRTM for evaluation of electromagnetic waves propagating along RRSs. It is shown that the DRTM is very effective to the field evaluation especially in a complicated environment, since it discretizes not only the terrain profile but also the procedure for searching rays, resulting in saving much computation time and computer memory. Section 4 discusses a numerical method to estimate propagation or path loss characteristics along 1D RRSs. An estimation formula for the radio communication distance along the 1D RRSs is also introduced in this Section. Section 5 is the conclusion of this chapter, and a few comments on the near future problems are remarked.

\section{Generation of 1D random rough surface}

As mentioned in the introduction, the sensor network has attracted many researchers' interest recently in different technical fields like signal processing, antennas, wave propagation, low power circuit design and so forth, just as the same as the case of radio frequency identification (RFID) [Heidrich,2010]. The sensor devices are usually located on terrestrial surfaces such as dessert, hilly terrain, forest, sea surface and so on. Since these surfaces are considered to be statistically random, it is important to study statistics of the RRSs as well as the electromagnetic wave propagation along them in order to construct reliable and efficient sensor network systems [Honda,2009].

In this section, we describe the statistical properties of RRSs and we show three types of spectral density functions, that is, Gaussian, $n$-th order of power-law and exponential spectra. We also discuss the convolution method for RRS generation. The convolution method is flexible and suitable for computer simulations to attack problems related to electromagnetic wave scattering from RRSs and electromagnetic wave propagation along RRSs.

\subsection{Spectral density function and auto-correlation function}

In this study, we assume that 1D RRSs extend in $x$-direction and it is uniform in $z$-direction with its height function as denoted by $y=f(x)$. The spectral density function $W(K)$ for a set of RRSs is defined by using the height function and the spatial angular frequency $K$ as follows:

$$
\int_{-\infty}^{\infty} W(K) d K=h^{2}
$$

where $h$ is the standard deviation of of the height function or height deviation, and

$$
W(K)=\lim _{L \rightarrow \infty} \frac{1}{2 \pi}\left\langle\frac{1}{L}\left|\int_{-L / 2}^{L / 2} f(x) e^{-j K x} d x\right|^{2}\right\rangle
$$


where $\langle>$ indicates the ensemble average of the RRS set. As is well-known, the autocorrelation function is given by the Fourier transform of the spectral density function as follows:

$$
\rho(x)=\int_{-\infty}^{\infty} W(K) e^{j K x} d K
$$

Now we summarize three types of spectral density functions that are useful for numerical simulations of the propagation characteristics of electromagnetic waves traveling along RRSs.

1. Gaussian Type of Spectrum:

The spectral density function of this type is defined by

$$
W(K)=\left(\frac{c l h^{2}}{2 \sqrt{\pi}}\right) e^{-\frac{K^{2} c l^{2}}{4}}
$$

where $\mathrm{cl}$ is the correlation length, and the auto-correlation function is given by

$$
\rho(x)=h^{2} e^{-\frac{x^{2}}{c l^{2}}} .
$$

2. $N$-th Order Power-Law Spectrum:

The spectral density function of this type is given by

$$
W(K)=\left(\frac{c l h^{2}}{2 \sqrt{\pi}}\right)\left\{1+\frac{\Gamma^{2}\left(N-\frac{1}{2}\right)}{\Gamma^{2}(N)} \frac{K^{2} c l^{2}}{4}\right\}^{-N}
$$

where $\Gamma(N)$ is the Gamma function with $N>1$, and the auto-correlation function is given by

$$
\rho(x)=\frac{h^{2}}{\left[1+\frac{x^{2}}{N c l^{2}}\right]^{N}} .
$$

3. Exponential Spectrum:

The spectral density function of this type is given by

$$
W(K)=\left(\frac{c l h^{2}}{\pi}\right)\left\{1+K^{2} c l^{2}\right\}^{-1}
$$

and the auto-correlation function is given by

$$
\rho(x)=h^{2} e^{-\frac{|x|}{c l}} .
$$

\subsection{Convolution method for RRS generation}

As is well-known, we should not use discrete Fourier transform (DFT) but fast Fourier transform (FFT) for practical applications to save computation time. For simplicity of analyses, however, we use DFT only for theoretical discussions. Now we consider a complex type of $1 \mathrm{D}$ array $\mathbf{f}$ corresponding to a discretized form of $f(x)$ and its complex type of spectral array F defined by

$$
\mathbf{f}=\left(f_{0}, f_{1}, f_{2}, \cdots, f_{N-2}, f_{N-1}\right), \mathbf{F}=\left(F_{0}, F_{1}, F_{2}, \cdots, F_{N-2}, F_{N-1}\right) .
$$


The complex type of spectral array $\mathbf{F}$ is the DFT of $\mathbf{f}$. And the complex type of DFT is defined as follows:

$$
\mathbf{F}=\operatorname{DFT}(\mathbf{f}), \quad F_{v}=\sum_{n=0}^{N-1} f_{n} e^{-j 2 \pi \frac{n v}{N}} \quad(v=0,1, \cdots, N-1) .
$$

Moreover, the inverse DFT is defined by

$$
\mathbf{f}=\mathrm{DFT}^{-1}(\mathbf{F}), f_{n}=\frac{1}{N} \sum_{v=0}^{N-1} F_{v} e^{j 2 \pi \frac{n v}{N}} \quad(n=0,1, \cdots, N-1) .
$$

First, we discretize the spectral density function discussed in the preceding section by introducing the discretized spatial angular frequency $K_{n}$ as follows:

$$
K_{n}=\frac{2 \pi n}{N_{1} c \ell} \quad(n=0,1,2, \cdots, N-1)
$$

where $N=N_{1} N_{2}$. It is assumed that $N_{2}$ is the number of discretized points per one correlation length $c l$ and correlation beyond the distance $N_{1} c l$ is negligibly small. Then we can obtain the real type of $1 \mathrm{D}$ array $\mathbf{w}$ by using the spectral density function $W(K)$ at the discretized spatial angular frequencies as follows:

$$
\mathbf{w}=\left(w_{0}, w_{1}, \cdots, w_{N-1}\right)
$$

where the elements $w_{n}$ of the array are expressed as follows:

$$
\begin{aligned}
& w_{n}=2 \pi W\left(K_{n^{\prime}}\right) / N_{1} c \ell \\
& n^{\prime}= \begin{cases}n & (0 \leq n<N / 2) \\
N-n & (N / 2 \leq n<N) .\end{cases}
\end{aligned}
$$

It should be noted that the DFT of the above 1D array corresponds to the discretized autocorrelation function of $\rho(x)$ as follows:

$$
\operatorname{DFT}(\mathbf{w}) \leftrightarrow \rho(x)
$$

Thus we can utilize this relation to check the accuracy of the discretized numerical results for the spectral density function of the RRSs we are dealing with.

Second, we introduce another real 1D array $\tilde{\mathbf{w}}$ by taking the square root of the former array as follows:

$$
\tilde{\mathbf{w}}=\left(\sqrt{w_{0} / N}, \sqrt{w_{1} / N}, \cdots, \sqrt{w_{N-1} / N}\right) .
$$

Performing the DFT of the above 1D array leads to a new weighting array defined by

$$
\tilde{\mathbf{W}}=\left(\tilde{W}_{0}, \tilde{W}_{1}, \cdots, \tilde{W}_{N-1}\right)=\operatorname{DFT}(\tilde{\mathbf{w}})
$$

This weighting array includes all the information about the spectral properties of the RRSs, and also it plays an important role as a weighting factor when we generate RRSs by the convolution method. 
Third, we consider the random number generator necessary for computer simulations. C programming language provides us the software rand $(a)$ which produces a sequence of random numbers ranging in $[0, a]$ [Johnsonbaugh,1997]. Then we can generate another sequence of random number $x_{i}$ in the following way:

$$
\begin{aligned}
& u_{1}=\operatorname{rand}(2 \pi), \quad u_{2}=\operatorname{rand}(1) \\
& x_{i}=\sqrt{-2 \log \left(u_{2}\right)} \cos \left(u_{1}\right) \quad(i=0,1,2, \ldots) .
\end{aligned}
$$

It can be proved that the random numbers obtained by the above functions belong to the normal distribution as follows:

$$
\left(x_{0}, x_{1}, x_{2}, \cdots\right) \in N(0,1)
$$

As a result, we can generate a sequence of the discrete random rough surface with arbitrary $N^{\prime}$ points by performing the discrete convolution between the sequence of the Gaussian random number $x_{i} \in N(1,0)$ given by Eq.19 and the weighting array $\tilde{W}_{k}$ given by Eq.18. The final results are summarized as follows:

$$
\begin{aligned}
& f_{n}=\sum_{k=0}^{N-1} \tilde{W}_{k} x_{n+k} \quad\left(n=0,1,2,3, \cdots, N^{\prime}-1\right) \\
& \left\{x_{i}\right\} \in N(1,0) \quad\left(i=0,1,2, \cdots, N+N^{\prime}-1\right) .
\end{aligned}
$$

Eq.21 is the essential part of the convolution method, and it provides us any type of RRSs with arbitrary length [Uchida,2007], [Uchida,2008].

It is worth noting that correlation of the generated RRSs is assumed to be negligibly small outside the distance of $N_{1} c l$ and the minimum discretized distance is $c \ell / N_{2}$. One of the advantages of the present convolution method is that we can generate continuous RRSs with an arbitrary number of sample points $N^{\prime}>N$, provided that the weighting array $\tilde{W}_{k}$ in Eq.18 is computed at the definite number of points $N=N_{1} N_{2}$. The other advantage is that the present method is more flexible and it saves more computation time than the conventional direct DFT method [Thoros,1989], [Thoros,1990], [Phu,1994], [Tsang,1994], [Yoon,2000], [Yoon,2002].

\section{Discrete ray tracing method (DRTM)}

In this chapter, we apply DRTM to the investigation of propagation characteristics along random rough surfaces whose height deviation $h$ and correlation length $c \ell$ are much longer than the wavelength, that is, $h, \mathrm{Cl}>>\lambda$. In the past, we used the ray tracing method (RTM) to analyze electromagnetic wave propagation along 1D RRSs. The RTM, however, requires lots of computer memory and computation time, since its ray searching algorithm is based only on the imaging method. The present DRTM, however, requires much less computer memory and computation time than the RTM. This is the reason why we employ the DRTM for ray searching and field computing. First, we discretize the rough surface in term of piecewiselinear lines, and second, we determine whether two lines are in the line of sight (LOS) or not (NLOS), depending on whether the two representative points on the two lines can be seen each other or not.

The field analyses of DRTM are based on the well-known edge diffraction problem by a conducting half plane which was rigorously solved by the Wiener-Hopf technique [Noble,1958]. The Wiener-Hopf solution cannot be rigorously applied to the diffraction problem by a plate 
of finite width. When the distance between the two edges of the plate is much longer than the wavelength, however, it can be approximately applied to this problem with an excellent accuracy. This is the basic idea of the field analyses based on DRTM. Numerical calculation are carried out for the propagation characteristics of electromagnetic waves traveling along RRSs with Gaussian, n-th order of power-law and exponential types of spectra.

\subsection{RRS discretization in terms of piecewise-linear profile}

A RRS of arbitrary length can be generated by the convolution method discussed in the preceding section. We treat here three types of spectral density functions for generating RRSs. The first is Gaussian, the second is n-th order of power-law and the third is exponential distribution, where the RRS parameters are correlation length $c l$ and height deviation $h$. Fig.1 shows four examples of RRSs with Gaussian, first and third order of power-law and exponential spectra, and the parameters are selected as $c l=10.0[\mathrm{~m}]$ and $h=1.0[\mathrm{~m}]$. It is shown that the Gaussian spectrum exhibits the smoothest roughness.

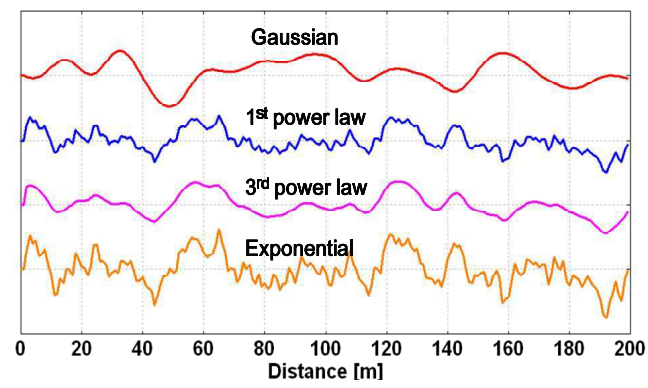

Fig. 1. Examples of random rough surface.

The convolution method introduced in the preceding section provides us the data of position vectors corresponding to the discretized RRS points as follows:

$$
\mathbf{r}_{\mathbf{n}}=\left(n \Delta x, f_{n}\right) \quad\left(n=0,1,2, \cdots, N^{\prime}-1\right)
$$

where the minimum discretized distance is given by

$$
\Delta x=c \ell / N_{2} .
$$

On the other hand, we can determine the normal vector of each straight line as follows:

$$
\mathbf{n}_{\mathbf{n}}=\left(\mathbf{u}_{\mathbf{z}} \times \mathbf{a}_{\mathbf{n}}\right) /\left|\mathbf{u}_{\mathbf{z}} \times \mathbf{a}_{\mathbf{n}}\right| \quad\left(n=0,1,2, \cdots, N^{\prime}-1\right)
$$

where $\mathbf{u}_{\mathbf{z}}$ is the unit vector in $z$-direction. Moreover, the vector corresponding to each straight line is given by

$$
\mathbf{a}_{\mathbf{i}}=\left(\mathbf{r}_{\mathbf{i}+\mathbf{1}}-\mathbf{r}_{\mathbf{i}}\right) .
$$

Thus all the informations regarding traced rays can be expressed in terms of the position vectors $\mathbf{r}_{\mathbf{n}}$ in Eq.22 and the normal vectors $\mathbf{n}_{\mathbf{n}}$ in Eq.24, resulting in saving computer memory. 


\subsection{Algorithm for searching rays based on DRTM}

Now we discuss the algorithm to trace discrete rays with respect to a discretized RRS. We propose a procedure to approximately determine whether the two straight lines $\mathbf{a}_{\mathbf{i}}$ and $\mathbf{a}_{\mathbf{j}}$ $(i \neq j)$ are in the line of sight (LOS) or not (NLOS) by checking whether the two representative points on the two lines can be seen from each other or not. The representative point of a line may be its center or one of its two edges, and in the following discussions, we employ the central point as a representative point of a line. Thus the essence of finding rays is reduced to checking whether the representative point of one straight line is in LOS or in NLOS of the representative point of the other line.

One type of ray is determined by constructing the minimum distance between the two representative points which are in NLOS, while the other type of ray is determined by connecting the two representative points which are in LOS. The traced rays obtained in this way are approximate, but the algorithm is simple and thus we can save much computation time. Moreover, we can modify the discrete rays into more accurate ones by applying the principle of the shortest path to the former case and the imaging method to the latter case. The former type is shown in (a) of Fig.2, and the latter type is depicted in (b) of Fig.2. In these figures, $S$ denotes a source point and $R$ indicates a receiver point.

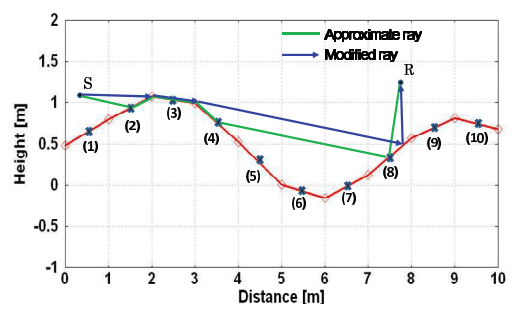

(a) in case of NLOS

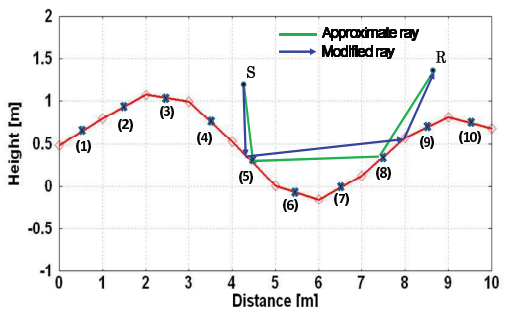

(b) in case of LOS

Fig. 2. Examples of searching rays.

Let us explain the example of searched ray in (a) of Fig.2. First we find a shortest path from (2) to (4) which are in NLOS, and we also find a straight line (4) to (8) which are in LOS. Moreover, we add the straight line from $S$ to (2) which are in LOS as well as the straight line from (8) to $R$ which are also in LOS. Thus we can draw an approximate discrete ray from $S$ to $R$ through (2), (3), (4) and (8). The discrete ray is shown by green lines. In order to construct a more accurate ray, we modify the discrete ray so that the distance from $S$ to (8) may be minimum, and we apply the imaging method to the discrete ray from (4) to $R$ through (8). The final modified ray is plotted in blue lines in (a) of Fig.2. The ray from $S$ to (8) constitutes a diffraction. We call it as a source diffraction, because it is associated with shadowing of the incident wave from source $S$ by the line (3).

Let us explain another example of searched ray shown in (b) of Fig.2. First, we find the straight line from (5) to (8) which are in LOS. Second, we add the lines from $S$ to (5) and from (8) to $R$, since $S$ and (5) as well as (8) and $R$ are in LOS. Thus we obtain an approximate discrete ray from $S$ to $R$ through (5) and (8) as shown in green lines. In order to obtain more accurate ray, we can modify the discrete ray based on the imaging method. The final ray plotted in blue lines shows that the ray emitted from $S$ is first reflected from the line at (5) and next diffracted at the right edge of the line at (8), and finally it reaches $R$. We call this type of diffraction as an 
image diffraction, since it is associated with reflection and the reflection might be described as an emission from the image point with respect to the related line.

\subsection{Reflection and diffraction coefficients}

The purpose of this investigation is to evaluate the propagation characteristics of electromagnetic waves traveling along RRSs from a source point $S$ to a receiver point $R$. We assume that the influences of transmitted waves through RRSs on propagation are negligibly small. As a result, the received electromagnetic waves at $R$ are expressed in terms of incident, reflected and diffracted rays in LOS region, and they are denoted in terms of reflected and diffracted rays in NLOS region.

First we consider electromagnetic wave reflection from a flat ground plane composed of a lossy dielectric. The lossy dielectric medium, for example, indicates a soil ground plane. Fig.3 shows a geometry of incidence and reflection with source point $S$ and receiver point $R$ together with the source's image point $S_{i}$. In Fig.3, the polarization of the incident wave is assumed such that electric field is parallel to the ground plane (z-axis) or magnetic field is parallel to it. We call the former case as E-wave or horizontal polarization, and we call the latter case as H-wave or vertical polarization [Mushuake,1985], [Collin,1985].

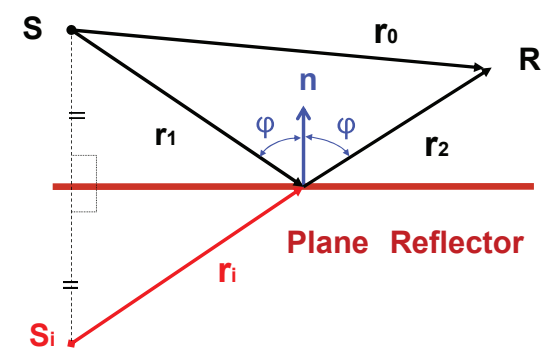

Fig. 3. Incidence and reflection.

The incident wave, which we also call a source field, and the reflected wave, which we also call an image field, are given by the following relations:

$$
E_{z}, H_{z}=\Psi\left(r_{0}\right)+R^{e, h}(\phi) \Psi\left(r_{1}+r_{2}\right)
$$

where $E_{z}$ and $H_{z}$ indicate E-wave (e) and $H$-wave (h), respectively. The distances $r_{0}, r_{1}$ and $r_{2}$ are depicted in Fig.3, and the complex field function expressing the amplitude and phase of a field is defined in terms of a propagation distance $r$ as follows:

$$
\Psi(r)=\frac{e^{-j k r}}{r} .
$$

In the field expressions, the time dependence $e^{j \omega t}$ is assumed and suppressed through out this chapter. The wavenumber $\kappa$ in the free space is given by

$$
\kappa=\omega \sqrt{\epsilon_{0} \mu_{0}}
$$


where $\epsilon_{0}$ and $\mu_{0}$ denote permittivity and permeability of the free space, respectively. $E_{z}$ and $H_{z}$ in Eq.26 correspond to $R^{e}(\phi)$ and $R^{h}(\phi)$, respectively. As mentioned earlier, E or H-wave indicates that electric or magnetic field is parallel to $z$-axis, respectively.

The reflection coefficients are expressed depending on the two different polarizations of the incident wave as follows:

$$
\begin{aligned}
& R^{e}(\phi)=\frac{\cos \phi-\sqrt{\epsilon_{\mathcal{C}}-\sin ^{2} \phi}}{\cos \phi+\sqrt{\epsilon_{\mathcal{C}}-\sin ^{2} \phi}} \\
& R^{h}(\phi)=\frac{\epsilon_{\mathcal{c}} \cos \phi-\sqrt{\epsilon_{c}-\sin ^{2} \phi}}{\epsilon_{\mathcal{C}} \cos \phi+\sqrt{\epsilon_{\mathcal{C}}-\sin ^{2} \phi}}
\end{aligned}
$$

where $\phi$ is the incident angle as shown in Fig.3. Moreover, the complex permittivity of the medium is given by

$$
\epsilon_{c}=\epsilon_{r}-j \frac{\sigma}{\omega \epsilon_{0}}
$$

where $\epsilon_{r}$ is dielectric constant and $\sigma$ is conductivity of the medium.

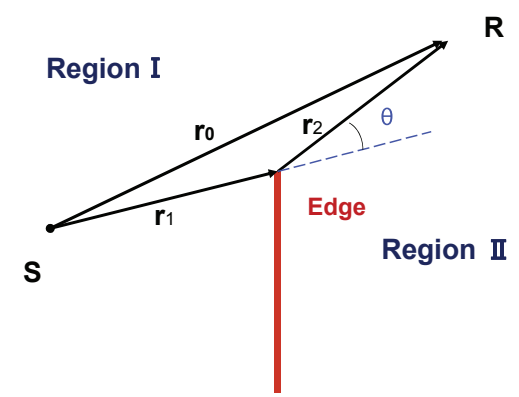

Fig. 4. Source diffraction from the edge of an half plane.

According to the rigorous solution for the plane wave diffraction by a half-plane [Noble,1958], diffraction phenomenon can be classified into two types. One is related to incident wave or field emitted from a source, which we call source field in short, as shown in Fig.4, and we call this type of diffraction as a source diffraction. The other is related to reflected wave or field emitted from an image, which we call image field in short, as shown in (a) of Fig.5, and we call this type of diffraction as an image diffraction. It should be noted that the rigorous solution based no the Wiener-Hopf technique is applicable only to the geometry of a semi-infinite half plane, and its extension to finite plate results in an approximate solution. However, it exhibits an excellent accuracy when the plate width is much longer than the wavelength. This is the starting point of the field analysis based on DRTM.

First we consider the source diffraction shown in Fig.4. In this case, we assume that the diffracted wave is approximated by the Winner-Hopf (WH) solution [Noble,1958]. The total diffracted fields for different two polarizations, that is $\mathrm{E}$ and $\mathrm{H}$-wave, are given by

$$
E_{z}, H_{z}= \begin{cases}D^{s} \Psi\left(r_{0}\right) & (\text { Region I) } \\ D^{s} \Psi\left(r_{1}+r_{2}\right) & (\text { Region II) }\end{cases}
$$


where the distances $r_{0}, r_{1}$ and $r_{2}$ are depicted in Fig. 4 and the complex field function is defined by Eq.27. The source diffraction coefficient is defined as follows:

$$
D^{s}= \begin{cases}1-D\left(r_{1}, r_{2}, \theta\right) \Psi\left(r_{1}+r_{2}\right) / \Psi\left(r_{0}\right) & (\text { Region I) } \\ D\left(r_{1}, r_{2}, \theta\right) & \text { (Region II) }\end{cases}
$$

where the diffraction function used in the above equation is defined by

$$
D\left(r_{1}, r_{2}, \theta\right)=e^{j X^{2}} F(X) .
$$

The complex type of Fresnel function for the diffraction function is defined by [Noble,1958]

$$
F(X)=\frac{e^{\frac{\pi}{4} j}}{\sqrt{\pi}} \int_{X}^{\infty} e^{-j u^{2}} d u \quad(X>0)
$$

where the argument is defined by

$$
X=\sqrt{\kappa\left(r_{1}+r_{2}-r_{0}\right)}
$$

and the distances $r_{0}, r_{1}$ and $r_{2}$ are shown in Fig.4.

Finally, we discuss the image diffraction as shown in (a) of Fig. 5 where $S$ is a source point, $S_{i}$ is its image point and $R$ is a receiver point, respectively. Two edges of the line of the dicretized rough surface are given by the position vectors defined by Eq.22 as follows:

$$
\mathbf{r}_{\mathbf{i} 1}=\left(i \Delta x, f_{i}\right), \quad \mathbf{r}_{\mathbf{i} 2}=\left((i+1) \Delta x, f_{i+1}\right) .
$$

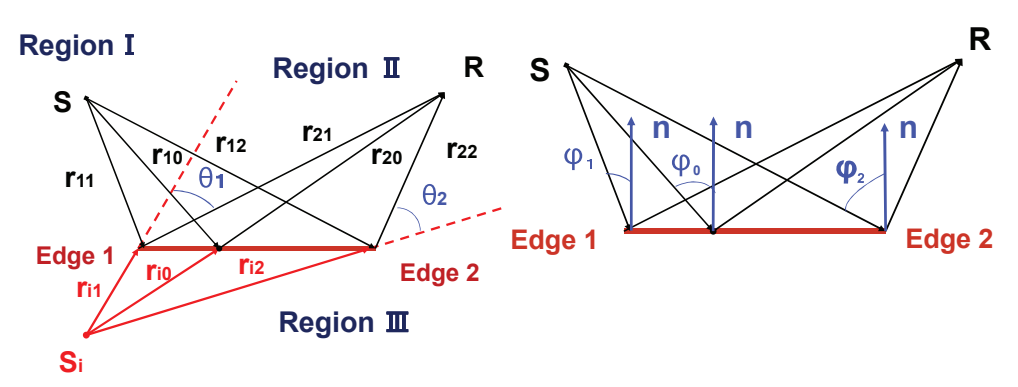

$\begin{array}{ll}\text { (a) Diffraction angles } \theta_{1} \text { and } \theta_{2} & \text { (b) Reflection angles } \phi_{1}, \phi_{2} \text { and } \phi_{0}\end{array}$

Fig. 5. Image diffraction.

Intersection between one straight line from $\mathbf{r}_{\mathbf{i} 1}$ to $\mathbf{r}_{\mathbf{i} 1}$ and the other straight line from the image point $S_{i}$ to the receiver point $R$ can be expressed as

$$
\mathbf{r}_{\mathbf{i} 0}=\mathbf{r}_{\mathbf{i} 1}+\eta\left(\mathbf{r}_{\mathbf{i} 2}-\mathbf{r}_{\mathbf{i} 1}\right)
$$

where $\eta$ is a constant to be determined. It is worth noting that the constant $\eta$ can be easily determined in terms of the position vectors, and $\eta<0$ AC $\leq \eta \leq 1$ and $1<\eta$ correspond to regions I, II and III in (a) of Fig.5, respectively. 
By use of the image diffraction coefficients as well as the field function in Eq.27, the image diffraction fields corresponding to the three regions are summarized as follows:

$$
E_{z}, H_{z}= \begin{cases}D^{i e, h} \Psi\left(r_{i 1}+r_{21}\right) & (\text { Rgion I) } \\ D^{i e, h} \Psi\left(r_{i 0}+r_{20}\right) & \text { (Region II) } \\ D^{i e, h} \Psi\left(r_{i 2}+r_{22}\right) & \text { (Region III) } .\end{cases}
$$

The image diffraction coefficients are expressed in terms of the reflection coefficients in Eq.29, the diffraction function in Eq.33 and the field function in Eq.27 as follows:

$$
D^{i e, h}=\left\{\begin{aligned}
& -R^{e, h}\left(\phi_{2}\right) D\left(r_{i 2}, r_{22}, \theta_{2}\right) \Psi\left(r_{i 2}+r_{22}\right) / \Psi\left(r_{i 1}+r_{21}\right) \\
& +R^{e, h}\left(\phi_{1}\right) D\left(r_{i 1}, r_{21}, \theta_{1}\right)(\text { RgionI }) \\
& -R^{e, h}\left(\phi_{1}\right) D\left(r_{i 1}, r_{21}, \theta_{1}\right) \Psi\left(r_{i 1}+r_{21}\right) / \Psi\left(r_{i 0}+r_{20}\right) \\
& -R^{e, h}\left(\phi_{2}\right) D\left(r_{i 2}, r_{22}, \theta_{2}\right) \Psi\left(r_{i 2}+r_{22}\right) / \Psi\left(r_{i 0}+r_{20}\right) \\
& +R^{e, h}\left(\phi_{0}\right)(\text { RegionII }) \\
& -R^{e, h}\left(\phi_{1}\right) D\left(r_{i 1}, r_{21}, \theta_{1}\right) \Psi\left(r_{i 1}+r_{21}\right) / \Psi\left(r_{i 2}+r_{22}\right) \\
& +R^{e, h}\left(\phi_{2}\right) D\left(r_{i 2}, r_{22}, \theta_{2}\right) \quad(\text { RegionIII })
\end{aligned}\right.
$$

where the two diffraction angles $\theta_{1}$ and $\theta_{1}$ are shown in (a) of Fig.5 and the three reflection angles $\phi_{0}, \phi_{1}$ and $\phi_{1}$ are depicted in (b) of Fig.5.

The main feature of the image diffraction is described in the following way by using the traced rays shown in (a) of Fig.5. In region I, we have two image diffraction rays $\left(S \rightarrow \mathbf{r}_{\mathbf{i 1}} \rightarrow R\right)$ and $\left(S \rightarrow \mathbf{r}_{\mathbf{i} 2} \rightarrow R\right)$. In region II we have two image diffraction rays $\left(S \rightarrow \mathbf{r}_{\mathbf{i} 1} \rightarrow R\right)$ and $\left(S \rightarrow \mathbf{r}_{\mathbf{i} 2} \rightarrow R\right)$ and a reflection ray $\left(S \rightarrow \mathbf{r}_{\mathbf{i} 0} \rightarrow R\right)$. In region III, we have two image diffraction rays $\left(S \rightarrow \mathbf{r}_{\mathbf{i} 1} \rightarrow R\right)$ and $\left(S \rightarrow \mathbf{r}_{\mathbf{i} 2} \rightarrow R\right.$ ). It should be noted that all these fields are continuous at the two boundaries of the three regions, that is, from I to II and from II to III.

\subsection{Field evaluations based on DRTM}

In the preceding sections, we have firstly proposed an algorithm to construct a discrete ray starting from a source, repeating source or image diffractions successively, and terminating at a receiver. Secondly, we have discussed the algorithm of DRTM to approximately evaluate the electromagnetic fields in relation to source or image diffractions based on the modified rays derived from the approximate discrete rays. As a result, electromagnetic fields along RRSs can be calculated numerically by repeating the DRTM computations step by step.

We assume that the source antenna is a small dipole antenna with gain $G=1.5$ [Mushiake,1985] and input power $P_{i}[W]$. The direction of the source antenna is denoted by a unit vector $\mathbf{p}$, and the $\mathrm{E}$ or $\mathrm{H}$-wave corresponds to the antenna direction parallel to $z$ or $y$-direction, respectively. The fields of the small dipole antenna are classified into three types, that is, static, induced and radiated fields. We call the first two terms as the near fields and the last one as the far field [Mushiuake,1985],[Collin,1985]. Contrary to RFID where the near fields are mainly used [Heidrich,2010], sensor networks mainly use the far fields which are predominant in the region where the distance from the source is much longer than the wavelength $(r>>\lambda)$.

Thus neglecting the near fields, the electric field radiating from a small dipole antenna is expressed in the following form [Mushiake,1985], [Collin,1985]

$$
\mathbf{E}_{\mathbf{0}}=\sqrt{30 G P_{i}}\left[\left(\mathbf{u}_{\mathbf{r}} \times \mathbf{p}\right) \times \mathbf{u}_{\mathbf{r}}\right] \Psi(r)
$$

where $\mathbf{r}$ is a position vector from the source to a receiver point and $r=|\mathbf{r}|$. The unit vector $\mathbf{u}_{\mathbf{r}}=\mathbf{r} / r$ is in the direction from the source to the receiver point, and $\left|\mathbf{u}_{\mathbf{r}} \times \mathbf{p}\right|=\sin \theta$ 
is the directivity of the small dipole antenna. The electric field radiated from the source antenna propagates along a RRS to a receiver point, decaying due to repeated source and image diffractions as discussed in the preceding section. We have assumed that the propagation model is $2 \mathrm{D}$, which means that the RRSs are uniform in $z$-direction and the direction of propagation is restricted only to the $(x, y)$-plane. This assumption indicates that the back and forward diffractions are predominant and the side diffractions are negligibly small, and this assumption might be valid as long as the isotropic 2D RRSs are concerned.

At a source diffraction point, the electric field is subject to both amplitude and phase conversions according to Eq.32, but this source diffraction gives rise to no conversion of polarization. At an image diffraction point, however, not only amplitude and phase conversions but also conversion of polarization occur. The latter conversion is described in such a way that E-wave conversion occurs for electric field component parallel to $z$-direction and $\mathrm{H}$-wave conversion does for electric field components perpendicular to $z$-direction as shown in Eq.39. At a source diffraction point, of course, the electric field receives the same conversion both for E-wave and H-wave as expressed in Eq.32.

Thus we can summarize the electric field at a receiver point in the following dyadic form

$$
\mathbf{E}=\sum_{n=1}^{N}\left[\prod_{m=1}^{m=M_{n}^{i}}\left(\mathbf{D}_{\mathbf{n m}}^{\mathbf{i}}\right) \cdot \prod_{k=1}^{k=M_{n}^{s}}\left(\mathbf{D}_{\mathbf{n k}}^{\mathbf{s}}\right) \cdot \mathbf{E}_{\mathbf{0}}\right] \Psi\left(r_{n}\right)
$$

where $\mathbf{E}_{0}$ is the electric field vector of the n-th ray at the first source or diffraction point. $N$ is the number of rays included for the field computations, $M_{n}^{s}$ is the number of source diffractions of the $n$-th ray, and $M_{n}^{i}$ is the number of its image diffractions. Moreover, $\mathbf{D}_{\mathbf{n k}}^{\mathbf{s}}$ is a dyadic source diffraction coefficient at the $k$-th source diffraction point of the $n$-th ray, and the coefficient can be computed by using the source diffraction coefficient $D^{s}$ defined in Eq.32. On the other hand, $\mathbf{D}_{\mathbf{n m}}^{\mathbf{i}}$ is a dyadic image diffraction coefficient at the $m$-th image diffraction point of the $n$-th ray, and the coefficient can be computed by using the image diffraction coefficients $D_{n m}^{i e}$ and $D_{n m}^{i h}$ defined in Eq.39. It should be noted that the source diffraction coefficient is the same both for $\mathrm{E}$ and $\mathrm{H}$-waves, while the image diffraction coefficient is different depending on the polarization of the fields at the image diffraction points. Moreover, the total distance of the $n$-th ray is given by

$$
r_{n}=\sum_{m=0}^{m=M_{n}^{s}+M_{n}^{i}} r_{n m} \quad(n=1,2, \ldots, N) .
$$

where $M_{n}^{s}$ and $M_{n}^{i}$ are the number of source and image diffraction points of the $n$-th ray, respectively.

It is worth noting that the specular reflection from a plate is included automatically as a special case of the image diffraction in the reflection region II of (a) in Fig.5. In the present DRTM computations, of course, the more the diffraction times increases, the more computation time is required. However, we can neglect the higher order of diffractions because their effects are small. In the present analyses, we include at most the three order of diffractions, resulting in saving much computation time compared to the method of moments (MoM) [Yagbasan,2010].

\section{Propagation characteristics of electromagnetic waves along RRSs}

In the preceding sections, we have proposed the convolution method for generating RRSs with two parameters, height deviation $h$ and correlation length $c l$. We have also proposed the 
DRTM to compute electric fields which are first radiated from a small dipole antenna, next propagate along RRSs repeating source and image diffractions, and finally arrive at a receiver. According to the DRTM process, the electromagnetic waves emitted from a source antenna propagate along RRSs, repeating reflection, diffraction and shadowing, and thus resulting in a more attenuation than in the free space.

The field distribution along one pattern of RRS exhibits one pattern of field variation with respect to propagating distance, and that of the other pattern of RRS shows another pattern of field variation. Accordingly every field distribution is different depending on the seed of RRS generation. However, as is evident from the theory of statistics, the ensemble average of the field distributions may show a definite propagation characteristics in a simple analytical form. This situation was empirically confirmed by Hata in case of the propagation characteristics in urban or suburban areas [Hata,1980].

\subsection{Distance characteristics of averaged field distribution}

Now we show a numerical example to explain the statistical properties of electromagnetic wave propagation along RRSs. In this numerical simulation, the source antenna is placed at $x=0[\mathrm{~m}]$ and at $0.5[\mathrm{~m}]$ high above RRSs, and the receiver point is movable along RRSs at $0.5[\mathrm{~m}]$ high above them. The operating frequency is chosen as $\mathrm{f}=1[\mathrm{GHz}]$. The RRS parameters are selected as height deviation $h=10[\mathrm{~m}]$ and correlation length $c l=50[\mathrm{~m}]$, and the material constants are chosen as dielectric constant $\epsilon_{r}=5$ and conductivity $\sigma=0.0023$ [S/m]. We assume here that the terrestrial ground is composed of a dry soil [Sato,2002].

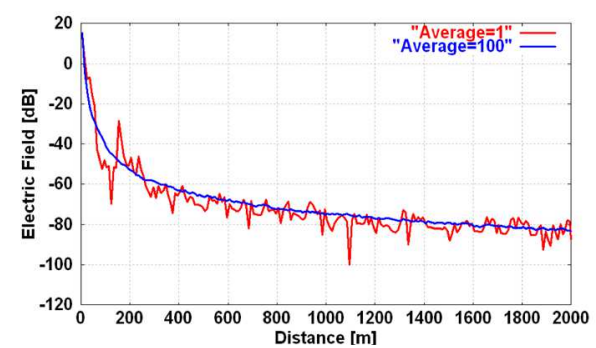

Fig. 6. Field distribution along one generated Gaussian RRS together with the ensemble average of 100 samples.

Fig.6 shows two electric field distributions along RRSs; one curve in red is the field distribution for one generated Gaussian RRS, and the other in blue is the ensemble average of the field distributions for 100 generated RRSs. In Fig.6, it is well demonstrated that one pattern of the field distributions is varying rapidly along the propagating distance, while the ensemble average of them is expressed in terms of a smooth and monotonic curve. As a result, it is concluded that we can approximate the ensemble average of the field distributions in terms of a simple analytic function.

Now we approximate the ensemble average of the electric field intensity by an analytic function with three constants, $\alpha, \beta$ and $\gamma$, as follows:

$$
E=\frac{10^{\frac{\alpha}{20}}}{(r+\gamma)^{\beta}}[V / m]
$$


where the unit input power or $P_{i}=1$ [W] in Eq.40 is assumed, and $\alpha$ is an amplitude weighting factor, $\beta$ is an order of propagation distance, and $\gamma$ is a distance correction factor. Rewriting the above relation in $\mathrm{dB}$ leads to the following equation [Hata,1980]:

$$
E=\alpha-20 \beta \log _{10}(r+\gamma)[d B] .
$$

Next, we determine the three unknown constants $\alpha, \beta$ and $\gamma$ based on the method of least mean square (LMS) with the objective function defined by

$$
\Phi(\alpha, \beta, \gamma)=\frac{1}{N} \sum_{n=1}^{N}\left(E_{n}^{d}-E_{n}\right)^{2} \longrightarrow \min
$$

where $N$ is the number of data. Moreover, $E_{n}^{d}$ is the averaged value of simulated or experimental data at $r=r_{n}$, and $E_{n}$ is the data computed by putting $r=r_{n}$ in Eq.43. Applying the conjugate gradient method (CGM) [Press,1992] to Eq.45, we can numerically determine the constants $\alpha, \beta$ and $\gamma$ explicitly.

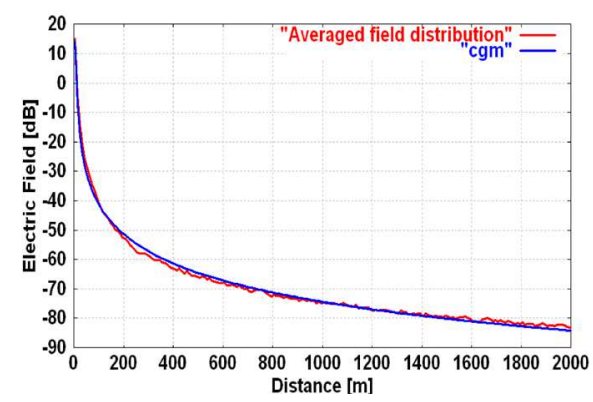

Fig. 7. Ensemble average of field distribution compared with estimated field distribution.

Fig.7 shows a comparison of the ensemble average of the field distribution with the estimated function based on the proposed algorithm. The estimated curve is given by Eq.43 with the constants determined by CGM such as $\alpha=22.6$ [dB], $\beta=1.62$ and $\gamma=-6.85$ [m]. It is worth noting that the distance correction factor $\gamma$ is small in comparison with the radio communication distance $r$, and thus we can neglect it as $\gamma \simeq 0$. Consequently, when we estimate the propagation characteristics of electromagnetic waves traveling along RRSs, the most important parameters are $\alpha$ and $\beta$ in Eq. 43 .

\subsection{Procedure for estimation of radio communication distance}

In the preceding section, we have demonstrated that the ensemble average of the field distributions of electromagnetic waves traveling along RRSs is well expressed in a simple and analytic function with three parameters $\alpha, \beta$ and $\gamma$. In this section, we propose an algorithm to estimate the radio communication distance when the two parameters for RRSs as well as another two parameters for source and receiver are specified. The first two parameters are the height deviation $h$ and the correlation length $c l$ for RRSs, and the last two parameters are the input power $P_{i}$ of a source antenna and the sensitivity of a receiver or the minimum detectable electric intensity $E_{m}$ of the receiver. 
Feasibility of the present method comes from the simplified form of the electric field distribution given by Eq.43. Once the three constants $\alpha, \beta$ and $\gamma$ are determined based on the simulated or experimental data, we can analytically evaluate the radio communication distance from a source with an arbitrary input power $P_{i}$ to a receiver with an arbitrary minimum detectable electric intensity $E_{\min }$. In this case we have assumed that both the source and receiver are on the homogeneous RRSs with the same parameters. Considering the $\mathrm{dB}$ expression such as $[\mathrm{dBm}]$ for the input power $P_{i}$ and $[\mathrm{dB} \mu \mathrm{V} / \mathrm{m}]$ for the minimum detectable electric intensity $E_{\text {min }}$, we can rewrite Eq.44 in the following form

$$
E_{\text {min }}-120=\alpha-20 \beta \log _{10}(r+\gamma)+P_{i}-30 .
$$

Solving for $r$ in the above equation, we can analytically determine the radio communication distance $r$ by the following relation:

$$
r=10^{\left(\alpha+P_{i}-E_{\min }+90\right) / 20 \beta}-\gamma \simeq 10^{\left(\alpha+P_{i}-E_{\min }+90\right) / 20 \beta} .
$$

Eq.47 is the estimation formula for the radio communication distance between the source antenna and receiver placed on homogeneous RRSs. The parameters of the RRSs are the height deviation $h[\mathrm{~m}]$ and correlation length $c \ell[\mathrm{m}]$. Moreover, the input power of the source is denoted by $P_{i}[\mathrm{dBm}]$ and the minimum detectable electric intensity of th receiver is described by $E_{\min }[\mathrm{dB} \mu \mathrm{V} / \mathrm{m}]$. As mentioned earlier, we can neglect the distance correction factor $\gamma$, and we have the more simplified estimation formula in Eq.47.

We show some numerical examples of the radio communication distances along RRSs computed by Eq.47. Fig.8 (a) shows radio communication distance $r$ [m] versus the minimum detectable electric field intensity $E_{\min }[\mathrm{dB} \mu \mathrm{V} / \mathrm{m}]$ of the receiver with the input power $P_{i}[\mathrm{dBm}]$ of the source antenna as a parameter. The height deviation of the RRSs is selected as $h=10[\mathrm{~m}]$ and the correlation length is chosen as $c l=50[\mathrm{~m}]$. The spectrum of the RRSs is assumed to be Gaussian, and the material constants of the RRS are chosen such that dielectric constant is $\epsilon_{r}=5$ and conductivity is $\sigma=0.023[\mathrm{~S} / \mathrm{m}]$. The operating frequency is chosen as $f=1[\mathrm{GHz}]$. The minimum detectable electric intensity $E_{\min }$ is varied from $40[\mathrm{~dB} \mu$ $\mathrm{V} / \mathrm{m}]$ to $80[\mathrm{~dB} \mu \mathrm{V} / \mathrm{m}]$, where the parameters for input power of source antenna are selected as $P_{i}=-10,-8, \cdots, 8,10[\mathrm{dBm}]$.

Fig.8 (b) shows radio communication distance $r[\mathrm{~m}]$ versus minimum detectable electric field intensity $E_{\min }[\mathrm{dB} \mu \mathrm{V} / \mathrm{m}]$ where the RRS height deviation is selected as $h=10[\mathrm{~m}]$ and its correlation length is chosen as $c l=100[\mathrm{~m}]$. Other parameters are chosen as the same values as in Fig.8 (a). In these two figures, it is demonstrated that the larger the input power of a source antenna becomes, the longer is the radio communication distance. It is also shown that the smaller the minimum detectable electric field intensity of a receiver becomes, the longer is the radio communication distance. Thus, based on the proposed procedure, we can estimate the radio communication distances along RRSs. 


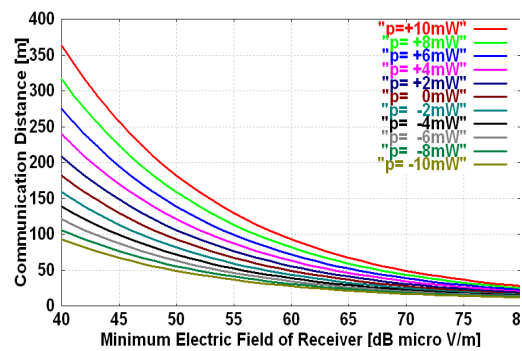

(a) $h=10 m$ and $c l=50 m$

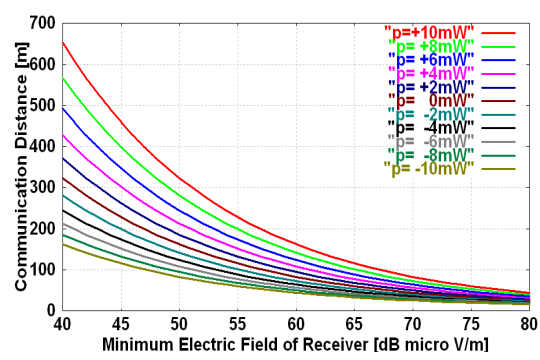

(b) $h=10 \mathrm{~m}$ and $c \ell=100 \mathrm{~m}$

Fig. 8. Radio communication distances along RRSs with Gaussian spectrum.

Fig.9 (a) and Fig.9 (b) show the radio communication distance versus minimum detectable electric intensity of a receiver located above the RRSs with the 1st order of power-law spectrum. The RRS parameters are chosen as the height deviation is $h=10[\mathrm{~m}]$ and the correlation length is $c l=50[\mathrm{~m}]$ in (a), and $h=10[\mathrm{~m}]$ and $c l=100[\mathrm{~m}]$ in (b), respectively. Other parameters are selected as the same as the former two Gaussian cases in Fig.8.

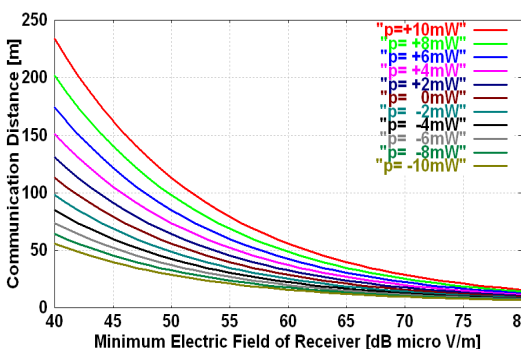

(a) $h=10 \mathrm{~m}$ and $c \ell=50 \mathrm{~m}$

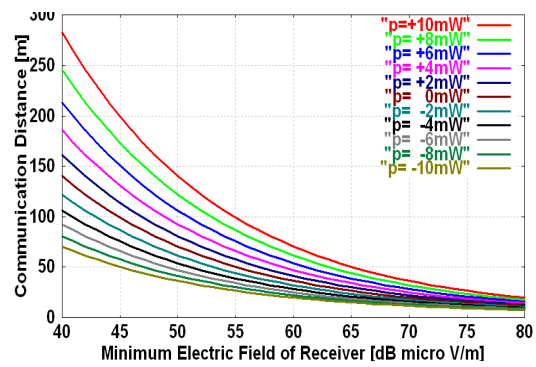

(b) $h=10 \mathrm{~m}$ and $c \ell=100 \mathrm{~m}$

Fig. 9. Radio communication distances along RRSs with power-law spectrum.

Fig.10 (a) and Fig.10 (b) show the radio communication distance versus minimum detectable electric intensity along the RRSs with exponential type of spectrum. The RRS parameters are chosen as $h=10[\mathrm{~m}]$ and $c l=50[\mathrm{~m}]$ in (a), and $h=10[\mathrm{~m}]$ and $c l=100[\mathrm{~m}]$ in (b), respectively. Other parameters are the same as the former examples in Figs. 8 and 9.

It is evident from the above numerical examples that the radio communication distances vary depending on the types of the RRS spectra, even though the RRS parameters $h$ and $c l$ are the same. It is shown that the radio communication distances along the RRSs with the Gaussian type of spectrum are the longest of the three types of spectra and those with the exponential type of spectrum are the shortest of the three. 


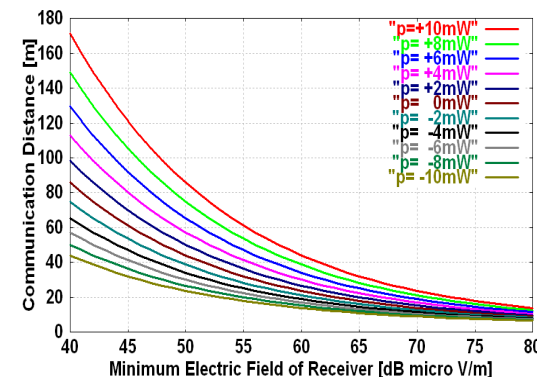

(a) $h=10 \mathrm{~m}$ and $c \ell=50 \mathrm{~m}$

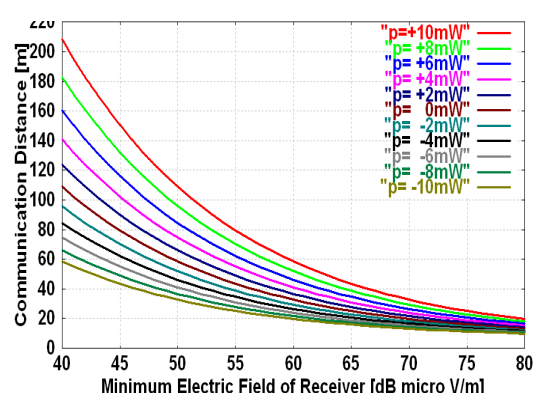

(b) $h=10 \mathrm{~m}$ and $c \ell=100 \mathrm{~m}$

Fig. 10. Radio communication distances along RRSs with exponential spectrum.

\section{Conclusion}

In this chapter, from a viewpoint of the application of radio communications to sensor networks, we have first discussed the convolution method to generate random rough surfaces (RRSs) to numerically simulate the propagation characteristics of electromagnetic waves traveling along the RRSs. Second, we have introduced the discrete ray tracing method (DRTM) to numerically evaluate the distributions of the electromagnetic waves along the RRSs. The remarkable point of the present method is to discretize not only the RRS's profile but also the ray tracing itself, resulting in saving much computation time. Third, we have proposed an algorithm to estimate the ensemble average of the field distributions in a simple and analytic expression by introducing the amplitude weighting factor $\alpha$, the order of propagation distance $\beta$ and the distance correction factor $\gamma$. Finally, we have introduced a formula to estimate the radio communication distance along RRSs, provided that the input power of a source antenna and the minimum detectable electric field intensity of a receiver are specified.

Numerical calculations were carried out for the dependence of radio communication distance versus minimum detectable electric field intensity of a receiver with the input power of a source antenna as a parameter. The results of computer simulations have revealed us that the longer the correlation length of the RRSs becomes, the longer are the radio communication distances. It has also been found that the radio communication distances along the RRSs vary depending on the types of spectra of the RRSs, and those of the Gaussian type of spectrum are longer than those of any other types of spectra.

We have treated only the homogeneous 1D RRSs to estimate the radio communication distances. In a more realistic situation, however, it is required to deal with inhomogeneous 2D RRSs [Uchida,2009]. This deserves as a near future investigation.

\section{References}

Aryanfar, F. \& Sarabandi, K. (2007). Validation of Wireless Channel Models Using a Scaled mm-Wave Measurement System, IEEE Antennas and Propagation Magazine, vol.49, no.4, pp.124-134.

Collin, R.E. (1985). Antennas and Radio Wave Propagation, McGraw-Hill Inc., New York, pp.1386.

Hata, M. (1980). Empirical formula for propagation loss in land mobile radio services, IEEE Trans. Veh. Technol., VT-29(3), pp.317-325. 
Heidrich, J. et al. (2010). The Roots, Rules, and Rise of RFID, IEEE Microwave Magazine, vol. 11 , no. 3 , pp.78-86.

Honda, J. et al. (2006). Effect of rough surface spectrum on propagation characteristics, IEEJ Technical Reports, EMT-06-128, pp.65-70.

Honda, J. \& Uchida, K. (2009). Discrete Ray-Tracing Method (DRTM) Analysis of Propagation Characteristics along Random Rough Surface in Relation to Development of Wireless Sensor Network, Proceedings of RWS 2009, TU2P-4, pp.248-251.

Honda, J., Uchida, K., Yoon, K.Y. (2010). Estimation of radio communication distance along random rough surface, IEICE Trans. Electron., E93-C(1), pp.39-45.

Johnsonbaugh, R. \& Kalin, M. (1997). C for Scientists and Engineers, Prentice-Hall, Inc., New Jersey, pp.191-195.

Mushiake, Y. (1985). Antennas and Radio Propagation, Corona Publishing Co., Ltd., p.39.

Noble, B. (1958). Methods based on the Wiener-Hopf technique, Pergamon Press.

Phu, P., Ishimaru, A., Kuga Y. (1994). Co-polarized and cross-polarized enhanced backscattering from two-dimensional very rough surfaces at millimeter wave frequencies, Radio Sci., 29(5), pp.1275-1291.

Press, W.H. et al. (1992). Numerical Recipes in Fortran 77: The Art of Scientific Computing, Cambridge University Press, New York, pp.387-448.

Sato, M. (2002). Subsurface imaging by ground penetrating radar, IEICE Trans. Electron., vol.J85-C, no.7, pp.520-530.

Thoros, E.I. (1988). The validity of the Kirchhoff approximation for rough surface scattering using a Gaussian roughness spectrum, J. Acoust. Soc. Am., 83(1), pp.78-92.

Thoros, E.I. (1990). Acoustic scattering from a Pierson-Moskowitz sea surface, J. Acoust. Soc. Am., 88(1), pp.335-349.

Tsang, L., Chan, C.H., Pak, K. (1994). Backscattering enhancement of a two-dimensional random rough surface (three-dimensional scattering) based on Monte Carlo simulation, J. Opt. Soc. Am. A, 11(2), pp.711-715.

Uchida, K. et al. (2007). FVTD analysis of electromagnetic wave propagation along random rough surface, IEICE Trans. Commun., J90-B(1), pp.48-55.

Uchida, K. et al. (2008).A@Analysis of electromagnetic wave propagation along rough surface by using discrete ray tracing method, Proceedings of ISAP 2008, pp.939-942.

Uchida, K., Honda, J., Yoon, K.Y. (2009). Distance characteristics of propagation in relation to inhomogeneity of random rough surface, Proceedings of ISMOT 2009, pp.1133-1136.

Yagbasan, A. et al. (2010). Characteristic Basis Function Method for solving Electromagnetic scattering Problems Over Rough Terrain Profiles, IEEE Trans. Antennas Propag., vol. 58 , no. 5 , pp. $1579-1589$.

Yoon, K.Y., Tateiba, M., Uchida, K. (2000). FVTD simulation for random rough dielectric surface scattering at low grazing angle, IEICE Trans. Electron., E83-C(12), 1836-1843.

Yoon, K.Y., Tateiba, M., Uchida, K. (2002). A numerical simulation of low-grazing-angle scattering from ocean-like dielectric surfaces, IEICE Trans. Commun., E85-B(10), pp.23442347. 


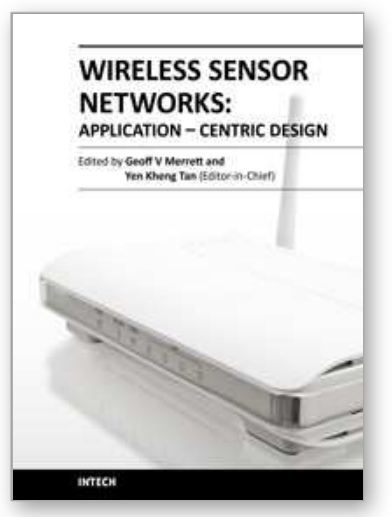

\author{
Wireless Sensor Networks: Application-Centric Design \\ Edited by Yen Kheng Tan
}

ISBN 978-953-307-321-7

Hard cover, 492 pages

Publisher InTech

Published online 14, December, 2010

Published in print edition December, 2010

Over the past decade, there has been a prolific increase in the research, development and commercialisation of Wireless Sensor Networks (WSNs) and their associated technologies. WSNs have found application in a vast range of different domains, scenarios and disciplines. These have included healthcare, defence and security, environmental monitoring and building/structural health monitoring. However, as a result of the broad array of pertinent applications, WSN researchers have also realised the application specificity of the domain; it is incredibly difficult, if not impossible, to find an application-independent solution to most WSN problems. Hence, research into WSNs dictates the adoption of an application-centric design process. This book is not intended to be a comprehensive review of all WSN applications and deployments to date. Instead, it is a collection of state-of-the-art research papers discussing current applications and deployment experiences, but also the communication and data processing technologies that are fundamental in further developing solutions to applications. Whilst a common foundation is retained through all chapters, this book contains a broad array of often differing interpretations, configurations and limitations of WSNs, and this highlights the diversity of this ever-changing research area. The chapters have been categorised into three distinct sections: applications and case studies, communication and networking, and information and data processing. The readership of this book is intended to be postgraduate/postdoctoral researchers and professional engineers, though some of the chapters may be of relevance to interested masterâ $€^{\mathrm{TM}} \mathrm{s}$ level students.

\title{
How to reference
}

In order to correctly reference this scholarly work, feel free to copy and paste the following:

Kazunori Uchida and Junichi Honda (2010). Estimation of Propagation Characteristics along Random Rough Surface for Sensor Networks, Wireless Sensor Networks: Application-Centric Design, Yen Kheng Tan (Ed.), ISBN: 978-953-307-321-7, InTech, Available from: http://www.intechopen.com/books/wireless-sensornetworks-application-centric-design/estimation-of-propagation-characteristics-along-random-rough-surfacefor-sensor-networks-

\section{INTECH}

open science | open minds

\section{InTech Europe}

University Campus STeP Ri

Slavka Krautzeka 83/A

51000 Rijeka, Croatia

\section{InTech China}

Unit 405, Office Block, Hotel Equatorial Shanghai

No.65, Yan An Road (West), Shanghai, 200040, China

中国上海市延安西路65号上海国际贵都大饭店办公楼405单元 
Phone: +385 (51) 770447

Fax: +385 (51) 686166

www.intechopen.com
Phone: +86-21-62489820

Fax: +86-21-62489821 
(C) 2010 The Author(s). Licensee IntechOpen. This chapter is distributed under the terms of the Creative Commons Attribution-NonCommercialShareAlike-3.0 License, which permits use, distribution and reproduction for non-commercial purposes, provided the original is properly cited and derivative works building on this content are distributed under the same license. 